\title{
Mortality, Morbidity, and Disease Severity of Patients with Aspiration Pneumonia
}

\author{
Michael J. Lanspa, MD,2*, Barbara E. Jones, MD¹, Samuel M. Brown, MD ${ }^{1,2}$, Nathan C. Dean, MD ${ }^{1,2}$
}

\begin{abstract}
${ }^{1}$ Division of Pulmonary and Critical Care Medicine, University of Utah School of Medicine, Salt Lake City, Utah; ${ }^{2}$ Division of Pulmonary and Critical
\end{abstract} Care Medicine, Intermountain Medical Center, Murray, Utah.

BACKGROUND: Aspiration pneumonia is a common syndrome, although less well characterized than other pneumonia syndromes. We describe a large population of patients with aspiration pneumonia.

METHODS: In this retrospective population study, we queried the electronic medical records at a tertiary-care, university-affiliated hospital from 1996 to 2006. Patients were initially identified by International Classification of Diseases, 9th Revision code 507.x; subsequent physician chart review excluded patients with aspiration pneumonitis and those without a confirmatory radiograph. Patients with community-acquired aspiration pneumonia were compared to a contemporaneous population of community-acquired pneumonia (CAP) patients. We compared CURB-65 (a clinical prediction rule based on Confusion, Uremia, Respiratory rate, Blood Pressure, and age)-predicted mortality with actual 30-day mortality.
RESULTS: We identified 628 patients with aspiration pneumonia, of which 510 were community-acquired. Median age was 77 years, with 30 -day mortality of $21 \%$. Compared to CAP patients, patients with community-acquired aspiration pneumonia had more frequent inpatient admission (99\% vs $58 \%$ ) and intensive care unit admission (38\% vs $14 \%$ ), higher Charlson comorbidity index (3 vs 1), and higher prevalence of do not resuscitate/intubate orders (24\% vs $11 \%$ ). CURB-65 predicted mortality poorly in aspiration pneumonia patients (area under the curve, 0.66).

CONCLUSIONS: Patients with community-acquired aspiration pneumonia are older, have more comorbidities, and demonstrate higher mortality than CAP patients, even after adjustment for age and comorbidities. CURB-65 poorly predicts mortality in this population. Journal of Hospital Medicine 2013;8:83-90. (c) 2012 Society of Hospital Medicine
Pneumonia is a common clinical syndrome with welldescribed epidemiology and microbiology. Aspiration pneumonia comprises $5 \%$ to $15 \%$ of patients with pneumonia, ${ }^{1}$ but is less well-characterized despite being a major syndrome of pneumonia in the elderly. ${ }^{2,3}$ Difficulties in studying aspiration pneumonia include the lack of a sensitive and specific marker for aspiration, the overlap between aspiration pneumonia and other forms of pneumonia, and the lack of differentiation between aspiration pneumonia and aspiration pneumonitis by many clinicians. ${ }^{4-6}$ Aspiration pneumonia, which develops after the aspiration of oropharyngeal contents, differs from aspiration pneumonitis, wherein inhalation of gastric contents causes inflammation without the subsequent development of bacterial infection. ${ }^{7,8}$

A number of validated mortality prediction models exist for community-acquired pneumonia (CAP), using a variety of clinical predictors. One clinical prediction

\footnotetext{
*Address for correspondence and reprint requests: Michael J. Lanspa, MD, Intermountain Medical Center, Shock-Trauma Intensive Care Unit, 5121 S. Cottonwood Street, Murray, UT 84107; Telephone: 801-507-6450; Fax: 801-507-4699; E-mail: michael.lanspa@imail.org Additional Supporting Information may be found in the online version of this article.
}

Received: May 15, 2012; Revised: October 9, 2012; Accepted: October 12, 2012

2012 Society of Hospital Medicine DOI 10.1002/jhm.1996

Published online in Wiley Online Library (Wileyonlinelibrary.com). rule endorsed by the British Thoracic Society is the CURB-65, which assigns a score for Confusion, Uremia $>19 \mathrm{mg} / \mathrm{dL}$, Respiratory rate $>=30$ breaths $/ \mathrm{min}$, Blood Pressure $<90 \mathrm{mmHg}$ systolic or $<60 \mathrm{mmHg}$ diastolic, and age $\geq 65$ ). We favor eCURB, a version of the CURB-65 model that uses continuously weighted variables to more accurately predict mortality, validated in CAP populations. ${ }^{9}$ Most studies validating pneumonia severity scoring systems excluded aspiration pneumonia from their study population. ${ }^{10-12}$ Severity scoring systems for CAP may not accurately predict disease severity patients with aspiration pneumonia.

The aims of our study were to: (1) identify a population of patients with aspiration pneumonia; (2) compare characteristics and outcomes in patients with community-acquired aspiration pneumonia to those with CAP; and (3) study the performance of eCURB and CURB-65 in predicting mortality for patients with community-acquired aspiration pneumonia.

\section{PATIENTS AND METHODS}

\section{Study Design and Setting}

The study was performed at LDS Hospital, a university-affiliated community teaching hospital in Salt Lake City, Utah, with 520 beds. In retrospective analysis of data from the electronic medical records, we identified all patients older than 18 years who were evaluated in the emergency department at LDS 


\begin{tabular}{ll}
\hline TABLE 1. Inclusion and Exclusion Criteria for the \\
Study \\
\hline Inclusion Criteria & Exclusion Criteria \\
\hline 1. Age $\geq 18$ years & $\begin{array}{l}\text { 1. Absence of radiographic evidence of } \\
\text { pneumonia within } 48 \text { hours after evaluation }\end{array}$ \\
$\begin{array}{ll}\text { 2. Either admitted to hospital or } & \text { 2. Previous episode of aspiration pneumonia } \\
\text { evaluated in emergency department } & \text { within } 12 \text { months } \\
\text { 3. 507.x code as primary diagnosis } & \text { 3. Initial admission date }>48 \text { hours before } \\
& \text { transfer to LDS Hospital } \\
\text { 4. } 507 . x \text { code as secondary diagnosis } & \text { 4. ADS } \\
\text { with a primary diagnosis of pneumonia, } & \text { 5. Receipt of antiretroviral therapy } \\
\text { respiratory failure, or septicemia } & \text { 6. History of solid organ transplant } \\
\text { 5. Treating physician indicated a } & \text { 7. Hematologic malignancy } \\
\text { diagnosis of aspiration pneumonia } & \text { 8. Witnessed isolated aspiration event within } \\
\text { in the history and physical and/or } & \text { 24 hours prior to evaluation } \\
\text { discharge summary } & \text { 9. Drug overdose, cardiopulmonary arrest, } \\
& \text { or seizure prior to hospital admission } \\
& \text { 10. Laryngoscopic or bronchoscopic evidence } \\
\text { of food material in airway }\end{array}$ \\
\hline
\end{tabular}

NOTE: Abbreviations: AIDS, acquired immune deficiency syndrome.

Hospital or admitted patients from other sources (direct admission, transfer from another hospital) from 1996 to 2006 with International Statistical Classification of Disease and Health Related Problems, 9th Revision (ICD-9) codes specific for aspiration pneumonia and pneumonitis (507.x). The treating physicians were mostly hospitalists and intensivists. Two physicians (M.L. and N.D.) manually reviewed the electronic medical records, including the emergency room physician's notes, the admission histories and physicals, the discharge summaries, and radiographic reports of the patients identified in the query. Consensus regarding the diagnosis of aspiration pneumonia was achieved in all patients reviewed using criteria listed in Table 1. This study was approved by the LDS Hospital institutional review board, and permission was granted to use the Utah Population Database for determining mortality (\#1008505), with a waiver of informed consent. For the contemporaneous group of CAP patients, we used a previously described population identified using ICD-9 codes 481.x to 487.x, captured from the same hospital during the same period. ${ }^{13}$

\section{Inclusion and Exclusion Criteria}

Inclusion and exclusion criteria are listed in Table 1 . To exclude patients with recurrent pneumonia, we included only the first episode of pneumonia in a given 12-month period. LDS Hospital frequently receives patients transferred from surrounding emergency departments and intensive care units. We excluded patients who were transferred $>48$ hours from their initial emergency department admission and therefore were late in their disease course. Exclusion criteria 8 to 10 were used to exclude patients with clinical presentations more consistent with aspiration pneumonitis. We also excluded immunocompromised patients (criteria 4 to 7 ).
TABLE 2. Minor Criteria for Severe CommunityAcquired Pneumonia, From the Infectious Disease Society of America/American Thoracic Society 2007 Criteria $^{17}$

Respiratory rate $\geq 30$ breaths/minute

$\mathrm{PaO}_{2} / \mathrm{FiO}_{2} \leq 250$

Multilobar infiltrates

Confusion/disorientation

Uremia (blood urea nitrogen $\geq 20 \mathrm{mg} / \mathrm{dL}$ )

Leukopenia (white blood cell count $<4000$ cells $/ \mathrm{mm}^{3}$ )

Thrombocytopenia (platelet count $<100,000$ cells $/ \mathrm{mm}^{3}$ )

Hypothermia (core temperature $\leq 36^{\circ} \mathrm{C}$ )

Hypotension requiring aggressive fluid resuscitation

Healthcare-associated aspiration pneumonia was defined as receipt of chronic hemodialysis, residence in a nursing facility, or hospitalization within any Intermountain Healthcare-affiliated hospital within the past 90 days. ${ }^{14}$ The remaining patients were defined as community-acquired aspiration pneumonia.

\section{Measurements}

The first vital signs, orientation status, and first 12 hours of routine laboratory results were extracted from the electronic medical records and used to calculate predicted mortality by eCURB and CURB-65. We determined 30-day mortality from the merger of the electronic medical records with vital status information from the Utah Population Database. ${ }^{15}$ The first measured $\mathrm{SpO}_{2}$ and $\mathrm{FiO}_{2}$ were used to estimate the $\mathrm{PaO}_{2} / \mathrm{FiO}_{2}$ ratio, using the Severinghaus calculation ${ }^{16}$ if no arterial blood gas was available. Presence of American Thoracic Society/Infectious Diseases Society of America (IDSA/ATS) 2007 minor criteria for severe community-acquired pneumonia $(\mathrm{SCAP})^{17}$ were obtained from baseline patient characteristics (Table 2). A Charlson comorbidity index was calculated from ICD-9 codes using published methodology. ${ }^{18,19}$ Presence of an abnormal swallow was defined as dysphagia or aspiration on modified barium swallow study, fiberoptic endoscopic evaluation, or clinical determination by a speech language pathologist during the index hospitalization. We also looked for causative pathogens, defined by a positive pneumococcus or legionella urinary antigen, or a positive culture from blood, bronchoalveolar lavage, pleural fluid, or tracheal aspirate, collected within 24 hours of admission. Antibiotics administered within the first 24 hours of admission were classified into 4 broad groups based on local physician prescribing patterns. Clindamycin and metronidazole were considered anaerobic-specific antibiotics. Vancomycin or linezolid were considered methicillin-resistant Staphylococcus aureus (MRSA) antibiotics. Broad-spectrum antibiotics included any of the following: carbapenems, aztreonam, piperacillin/tazobactam, ticarcillin/clavulanate, cefepime, and ceftazidime. Macrolides, respiratory fluoroquinolones, 


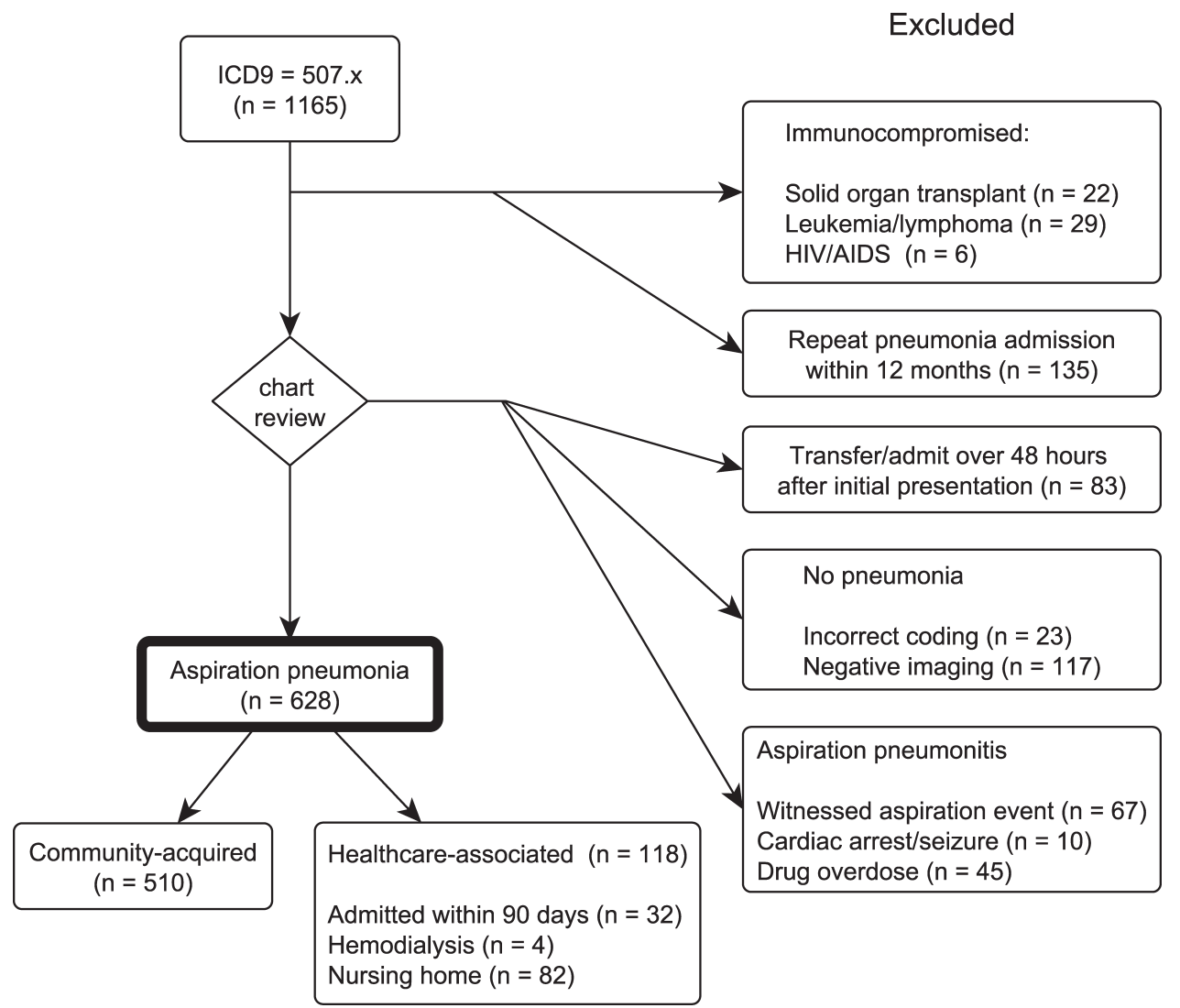

FIG. 1. Inclusion and exclusion criteria. Abbreviations: HIV/AIDS, human immunodeficiency virus/acquired immune deficiency syndrome; ICD-9, International Classification of Diseases, 9th Revision.

and third-generation cephalosporins were considered standard-care antibiotics.

\section{Statistical Analysis}

We compared baseline patient characteristics and clinical outcomes using the Fisher exact test to compare proportions of categorical variables, and Mann-Whitney $U$ test or Student $t$ test to compare central tendencies of continuous variables, as dictated by the normality of the data. Receiver operating characteristic curves calculated the ability of eCURB and CURB-65 to predict 30-day mortality prediction in patients with communityacquired aspiration pneumonia and CAP, as well as the ability of IDSA/ATS minor criteria for SCAP to predict admission to the intensive care unit (ICU). We performed multivariate logistic regression to predict 30-day mortality in patients with community-acquired aspiration pneumonia and CAP, using stepwise backward elimination. Confounders were included if they were significant at a 0.05 level or if they altered the coefficient of the main variable by more than $10 \%$. For logistic models, we evaluated goodness of fit with the Hosmer-Lemeshow technique; comparisons of area under the curve (AUC) among models were made using the technique of DeLong. ${ }^{20}$ Two-tailed $P$ values of $\leq 0.05$ were considered statistically significant. Stata version 12 (StataCorp, College Station, TX) was used for all analyses.

\section{RESULTS}

Our initial query identified 1165 patients. Physician review of the medical records resulted in 628 patients, 118 of whom were classified as healthcare-associated aspiration pneumonia (Figure 1, Table 3). Of all aspiration pneumonia patients, $80 \%$ were seen in the emergency department, $12.5 \%$ were directly admitted from the community, and $7.5 \%$ were transferred from another healthcare facility. Almost all patients seen in the emergency department $(99.0 \%)$ were admitted to the hospital, with median length of hospitalization 6.7 days among survivors.

Observed mortality was $21.0 \%$. eCURB significantly underestimated mortality in this group, predicting a mortality rate of $10.6 \%$. When classifying patients by the 2007 IDSA/ATS guidelines, $24.7 \%$ of the patients had 3 or more minor criteria for SCAP. ${ }^{17}$ The $\mathrm{PaO}_{2}$ / $\mathrm{FiO}_{2}$ ratio was obtained in $99.7 \%$ of patients. The median $\mathrm{PaO}_{2} / \mathrm{FiO}_{2}$ ratio observed in this population was $221 \mathrm{~mm} \mathrm{Hg}$ (equivalent to $260 \mathrm{~mm} \mathrm{Hg}$ at sea level barometric pressure, adjusted for our altitude of 1400 meters), near the threshold sea level definition $(250 \mathrm{~mm} \mathrm{Hg})$ for SCAP. ${ }^{13,17}$ Admission to the ICU was common, as were admission orders for "do not resuscitate" (DNR) or "do not intubate" (DNI). Patients with healthcare-associated aspiration pneumonia had a higher comorbidity index and had a 
TABLE 3. Patient Characteristics of Aspiration Pneumonia, Subdivided by Presence of Healthcare Association

\begin{tabular}{|c|c|c|c|c|}
\hline & $\begin{array}{l}\text { Aspiration } \\
\text { Pneumonia }(\mathrm{N}=628)\end{array}$ & $\begin{array}{l}\text { Community-Acquired } \\
\text { Aspiration Pneumonia ( } N=510)\end{array}$ & $\begin{array}{l}\text { Healthcare Associated } \\
\text { Aspiration Pneumonia }(\mathrm{N}=118)\end{array}$ & $P$ Value \\
\hline Age (range), y & $77(65-85)$ & $77(64-85)$ & $80(67-86)$ & 0.42 \\
\hline Female, $\%$ & 49.8 & 50.2 & 48.3 & 0.76 \\
\hline 30-day mortality, \% & $21.0 \%$ & $19.0 \%$ & $29.7 \%$ & 0.02 \\
\hline CURB-65 score & $2(1-3)$ & $2(1-3)$ & $2(1-3)$ & 0.0012 \\
\hline Confusion & $13.9 \%$ & $12.7 \%$ & $18.6 \%$ & 0.10 \\
\hline Blood urea nitrogen (mg/dL) & $22(16-34)$ & $21(15-32)$ & $30(20-47)$ & $<0.0001$ \\
\hline Respiratory rate (breaths/min) & $20(18-26)$ & $20(18-24)$ & $21(18-28)$ & 0.30 \\
\hline Systolic blood pressure (mm Hg) & $128(110-149)$ & $129(110-150)$ & $127(105-146)$ & 0.28 \\
\hline eCURB 30-day mortality estimate (median, \%) & $5.6(2.4-14.2)$ & $5.2(2.2-12.4)$ & $8.9(4.2-22.5)$ & $<0.0001$ \\
\hline eCURB 30-day mortality estimate (mean, \%) & $10.6 \pm 12.2$ & $9.7 \pm 11.5$ & $14.6 \pm 14.1$ & $<0.0001$ \\
\hline Hospital admission (of ED visits), \% & 99.0 & 98.8 & 100 & 0.59 \\
\hline Hospital LOS, d & $6.7(4.1-11.1)$ & $6.5(4.0-11.0)$ & $7.8(5.4-12.3)$ & 0.05 \\
\hline ICU admission, \% & 37.9 & 37.1 & 41.5 & 0.21 \\
\hline ICU LOS, d & $3.5(1.9-8.8)$ & $3.1(1.8-7.6)$ & $5.6(3.8-10.8)$ & 0.02 \\
\hline Mean ventilator-free days (of ICU patients, out of 30 days) & $25.2 \pm 8.3$ & $25.9 \pm 7.7$ & $22.7 \pm 10.0$ & 0.01 \\
\hline Receipt of mechanical ventilation, $\%$ & 18.6 & 17.2 & 24.6 & 0.09 \\
\hline Duration of ventilation, $d$ & $2.8(0.9-6.5)$ & $3.1(1.0-6.6)$ & $1.9(0.8-6.3)$ & 0.05 \\
\hline Receipt of vasopressor, \% & 1.8 & 1.4 & 3.4 & 0.13 \\
\hline Charlson comorbidity index & $4(2-6)$ & $3(2-6)$ & $4(3-6)$ & 0.0024 \\
\hline Cerebrovascular disease, \% & 33.9 & 32.4 & 40.7 & 0.11 \\
\hline Chronic pulmonary disease, $\%$ & 51.0 & 51.8 & 47.5 & 0.42 \\
\hline Congestive heart failure, \% & 52.4 & 50.0 & 62.7 & 0.01 \\
\hline Connective tissue disease, \% & 8.4 & 8.8 & 6.8 & 0.58 \\
\hline Dementia, \% & 14.2 & 12.0 & 23.7 & 0.0019 \\
\hline Hemiplegia/paraplegia & 9.4 & 8.0 & 15.2 & 0.02 \\
\hline Myocardial infarction, \% & 21.0 & 17.8 & 29.7 & 0.02 \\
\hline Peripheral vascular disease, \% & 17.7 & 16.3 & 23.7 & 0.06 \\
\hline Peptic ulcer disease, \% & 18.8 & 19.2 & 16.9 & 0.70 \\
\hline Diabetes without complications, \% & 10.7 & 9.2 & 16.9 & 0.02 \\
\hline Diabetes with complications, \% & 31.5 & 30.4 & 36.4 & 0.23 \\
\hline Mild liver disease, \% & 8.6 & 8.0 & 11.0 & 0.28 \\
\hline Moderate or severe liver disease, \% & 1.8 & 1.6 & 2.5 & 0.44 \\
\hline Malignant solid tumor, \% & 16.6 & 17.3 & 13.6 & 0.41 \\
\hline Metastatic cancer, \% & 5.4 & 5.7 & 4.2 & 0.66 \\
\hline Renal disease, \% & 14.7 & 4.2 & 18.6 & 0.19 \\
\hline 3 or more minor SCAP criteria, $\%{ }^{*}$ & 24.7 & 23.1 & 31.4 & 0.08 \\
\hline $\mathrm{PaO}_{2} / \mathrm{FiO}_{2}$ ratio & $221(161-280)$ & $226(169-280)$ & $181(133-245)$ & 0.0004 \\
\hline Multilobar disease, \% & 46.3 & 43.2 & 53.9 & 0.11 \\
\hline Presence of an effusion, $\%$ & 23.1 & 19.7 & 31.9 & 0.03 \\
\hline Swallow impairment (of tested survivors), \% & 34.1 & 34.1 & 34.1 & 0.22 \\
\hline Presence of a DNR/DNI order, \% & 26.4 & 23.7 & 38.1 & 0.0024 \\
\hline Mortality of patients with DNR/DNI order, $\%$ & 39.1 & 38.8 & 40.0 & 1.00 \\
\hline Receipt of broad-spectrum antibiotic, \% & 35.4 & 32.5 & 47.5 & 0.0028 \\
\hline Receipt of MRSA antibiotic, \% & 7.5 & 5.7 & 15.3 & 0.0014 \\
\hline Receipt of anaerobe antibiotic, \% & 28.7 & 27.6 & 33.1 & 0.26 \\
\hline
\end{tabular}

NOTE: All continuous or ordinal data are median values followed by interquartile ranges, unless otherwise specified. Significance testing between community-acquired aspiration pneumonia and healthcare-associated aspiration pneumonia was calculated with Fisher exact or Wilcoxon tests, where appropriate. Abbreviations: AUC, area under the curve; DNR/DNI, Do not resuscitate/do not intubate; ED, emergency department; LOS, length of stay; MRSA, methicillin-resistant Staphylococcus aureus; SCAP, severe community-acquired pneumonia. *SCAP described in the 2007 Infectious Diseases Society of America/American Thoracic Society guidelines (Table 2).

higher mortality rate than patients with communityacquired aspiration pneumonia, although we found no significant difference in the rate of hospital or ICU admission or the receipt of critical care therapies. Inpatient assessment of dysphagia and aspiration was conducted in 177 patients. Abnormal swallow was noted in $96 \%$ of those tested.

We found several differences between patients with community-acquired aspiration pneumonia and 2584 patients with CAP identified during the same time pe$\operatorname{riod}^{13}$ (Table 4). Patients with community-acquired aspiration pneumonia were older, more likely to have multilobar disease or effusion on imaging, and had greater disease severity. They also had a higher frequency of ICU and hospital admission, IDSA/ATS minor criteria for SCAP, and higher Charlson comorbidity indices. Patients with community-acquired aspiration pneumonia were more likely to receive mechanical ventilation than CAP patients, although there was no difference in 30-day mortality among intubated patients or a difference in ventilator-free days.

Thirty-day mortality for patients with communityacquired aspiration pneumonia was significantly higher than in CAP patients. Patients with 
TABLE 4. Comparison of Community-Acquired

Aspiration Pneumonia and Typical

Community-Acquired Pneumonia

\begin{tabular}{|c|c|c|c|}
\hline & $\begin{array}{l}\text { Community-Acquired } \\
\text { Aspiration } \\
\text { Pneumonia } \\
(\mathrm{N}=510)\end{array}$ & $\begin{array}{l}\text { Community- } \\
\text { Acquired } \\
\text { Pneumonia } \\
(\mathrm{N}=2584)\end{array}$ & $P$ Value \\
\hline Age (range), y & $77(64-85)$ & $59(41-76)$ & $<0.0001$ \\
\hline Female, \% & 50.2 & 49.5 & 0.81 \\
\hline 30-day mortality, \% & 19.0 & 4.2 & $<0.0001$ \\
\hline CURB-65 score & $2(1-3)$ & $1(0-2)$ & $<0.0001$ \\
\hline Confusion, \% & 12.7 & 5.1 & $<0.0001$ \\
\hline Blood urea nitrogen & $21(15-32)$ & $16(11-24)$ & $<0.0001$ \\
\hline Respiratory rate & $20(18-24)$ & $20(18-24)$ & $<0.0001$ \\
\hline Systolic blood pressure & $129(110-150)$ & $130(112-146)$ & 0.67 \\
\hline $\begin{array}{l}\text { eCURB 30-day mortality } \\
\text { estimate, median, \% }\end{array}$ & $5.2(2.2-12.4)$ & $1.7(0.9-4.3)$ & $<0.0001$ \\
\hline $\begin{array}{l}\text { eCURB 30-day mortality } \\
\text { estimate, mean, \% }\end{array}$ & $9.7 \pm 11.5$ & $4.4 \pm 7.5$ & $<0.0001$ \\
\hline AUC of eCURB versus mortality & $0.71(0.66-0.75)$ & $0.86(0.83-0.90)$ & $<0.0001$ \\
\hline Excluding DNR/DNI patients & $0.69(0.65-0.74)$ & $0.87(0.83-0.90)$ & 0.0001 \\
\hline AUC of CURB-65 versus mortality & $0.66(0.62-0.69)$ & $0.81(0.78-0.85)$ & $<0.0001$ \\
\hline Excluding DNR/DNI patients & $0.65(0.60-0.70)$ & $0.81(0.76-0.85)$ & 0.0003 \\
\hline Hospital admission (of ED visits), \% & 98.8 & 57.8 & $<0.0001$ \\
\hline Hospital LOS, d & $6.5(4.0-11.0)$ & $3.3(2.2-5.2)$ & $<0.0001$ \\
\hline ICU admission, \% & 37.1 & 14.2 & $<0.0001$ \\
\hline ICU LOS, d & $3.1(1.8-7.6)$ & $2.5(1.1-7.7)$ & 0.01 \\
\hline $\begin{array}{l}\text { Mean ventilator-free days (of ICU } \\
\text { patients, out of } 30 \text { days) }\end{array}$ & $25.9 \pm 7.7$ & $25 \pm 9$ & 0.75 \\
\hline Receipt of mechanical ventilation, \% & 17.2 & 7.8 & $<0.0001$ \\
\hline Duration of ventilation, $d$ & $3.1(1.0-6.6)$ & $3.5(1.5-7.2)$ & 0.09 \\
\hline Receipt of vasopressor, \% & 1.4 & 3.3 & 0.02 \\
\hline Charlson comorbidity index & $3(2-6)$ & $1(0-3)$ & $<0.0001$ \\
\hline Cerebrovascular disease, \% & 32.4 & 10.0 & $<0.0001$ \\
\hline Chronic pulmonary disease, $\%$ & 51.8 & 42.5 & $<0.0001$ \\
\hline Congestive heart failure, $\%$ & 50.0 & 22.1 & $<0.0001$ \\
\hline Connective tissue disease, \% & 8.8 & 5.6 & 0.0084 \\
\hline Dementia, \% & 12.0 & 2.8 & $<0.0001$ \\
\hline Hemiplegia/paraplegia, \% & 8.0 & 2.7 & $<0.0001$ \\
\hline Myocardial infarction, \% & 17.8 & 10.8 & $<0.0001$ \\
\hline Peripheral vascular disease, \% & 16.3 & 7.4 & $<0.0001$ \\
\hline Peptic ulcer disease, \% & 19.2 & 7.6 & $<0.0001$ \\
\hline Diabetes without complications, \% & 9.2 & 24.7 & $<0.0001$ \\
\hline Diabetes with complications, \% & 30.4 & 5.1 & $<0.0001$ \\
\hline Mild liver disease, $\%$ & 8.0 & 6.2 & 0.14 \\
\hline Moderate or severe liver disease, \% & 1.6 & 0.8 & 0.13 \\
\hline Malignant solid tumor, \% & 17. & 8.9 & $<0.0001$ \\
\hline Metastatic cancer, \% & 5.7 & 1.3 & $<0.0001$ \\
\hline Renal disease, \% & 4.2 & 5.6 & $<0.0001$ \\
\hline 3 or more minor SCAP criteria, $\%{ }^{*}$ & 24.7 & 19.1 & 0.01 \\
\hline $\mathrm{PaO}_{2} / \mathrm{FiO}_{2}$ ratio & $226(169-280)$ & $260(148-338)$ & 0.0004 \\
\hline Multilobar disease, \% & 43.2 & 37.2 & 0.0012 \\
\hline Presence of an effusion, $\%$ & 19.7 & 18.3 & $<0.0001$ \\
\hline Presence of a DNR/DNI order, \% & 23.7 & 9.7 & $<0.0001$ \\
\hline $\begin{array}{l}\text { Mortality of patients with } \\
\text { DNR/DNl order, } \%\end{array}$ & 38.8 & 12.4 & $<0.0001$ \\
\hline $\begin{array}{l}\text { Receipt of broad-spectrum } \\
\text { antibiotic, \% }\end{array}$ & 32.5 & 8.4 & $<0.0001$ \\
\hline Receipt of MRSA antibiotic, \% & 5.7 & 2.2 & $<0.0001$ \\
\hline Receipt of anaerobe antibiotic, \% & 27.6 & 3.1 & $<0.0001$ \\
\hline
\end{tabular}

NOTE: All dichotomous data are proportions. All continuous or ordinal data are median values followed by interquartile ranges, unless otherwise specified. Significance testing was calculated with Fisher exact or Wilcoxon tests, where appropriate. Abbreviations: AUC, area under the curve; CURB-65, a clinical prediction rule based on Confusion, Uremia, Respiratory rate, Blood Pressure, and age > 65; DNR/DNI, do not resuscitate/do not intubate; CCURB, a version of the CURB-65 mode that uses continuously weighted variables: ED, emergency department; ICU, intensive care unit; LOS, length of stay; MRSA, methicillin-resistant Staphylococcus aureus; SCAP, severe community-acquired pneumonia *SCAP described in the 2007 Infectious Diseases Society of America/American Thoracic Society guidelines.

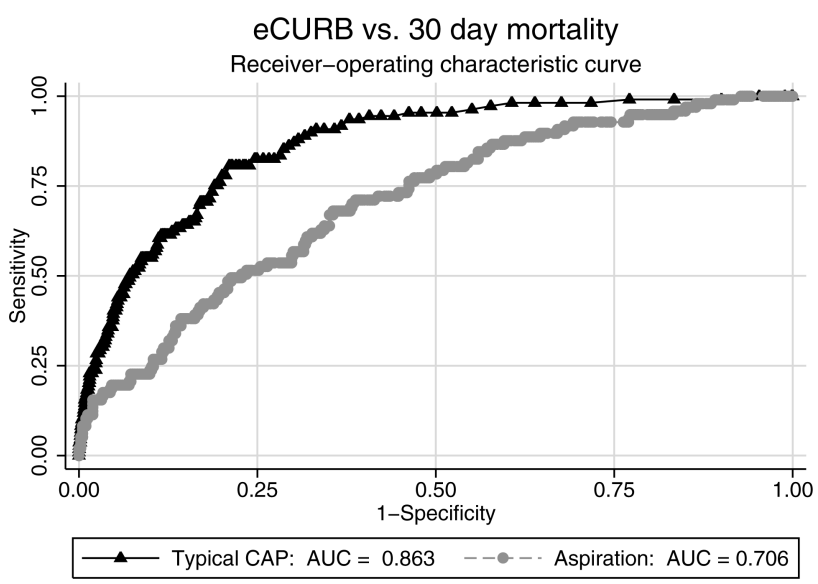

FIG. 2. Receiver operating characteristic curve, comparing the eCURB score against 30-day mortality in patients with typical community-acquired pneumonia and in patients with community-acquired aspiration pneumonia. The eCURB score is an electronic version of the CURB-65 model, validated in the community-acquired pneumonia population, that uses continuously weighted variables to more accurately predict mortality. These curves statistically differ, $P<0.0001$. Abbreviations: AUC, area under the curve; CAP, community-acquired pneumonia.

IDSA/ATS minor criteria for severe CAP vs. ICU admission Receiver-operating characteristic curve

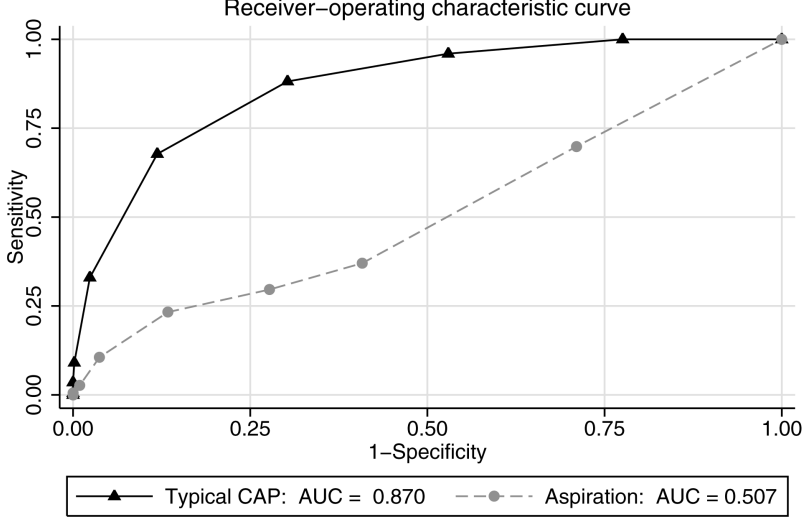

FIG. 3. Receiver operating characteristic curve, comparing the Infectious Diseases Society of America/American Thoracic Society (IDSA/ATS) minor criteria for severe community-acquired pneumonia against intensive care unit (ICU) admission in patients with typical community-acquired pneumonia (CAP) and in patients with community-acquired aspiration pneumonia. These curves statistically differ, $P<0.0001$. Abbreviations: AUC: area under the curve.

community-acquired aspiration pneumonia also had higher eCURB and CURB-65 scores. However, eCURB was a poor predictor of 30-day mortality, with an AUC of 0.71 , compared to 0.86 calculated for the CAP population (Figure 2). CURB-65 performed similarly: AUC was 0.66 vs 0.81 . The presence of a DNR/DNI order was twice as prevalent in the community-acquired aspiration pneumonia population vs the CAP population; those patients with a DNR/ DNI order were 3 times as likely to die. Excluding patients with a DNR/DNI order did not improve performance of eCURB or CURB-65 (Table 4). The presence of IDSA/ATS minor criteria for SCAP was not predictive of triage to the ICU in the group of patients 
with community-acquired aspiration pneumonia (AUC: 0.51), compared with CAP patients (AUC: $0.88, P<0.01$ for comparison, Figure 3$)$. This finding persisted in the subset of patients without a DNR/ DNI order (AUC: 0.52 in community-acquired aspiration pneumonia vs 0.88 in CAP, $P<0.01$ ).

Our regression model of mortality incorporated gender, presence of effusion or multilobar pneumonia, presence of a DNR/DNI order, and all the components of the CURB-65, IDSA/ATS minor criteria for SCAP, and Charlson comorbidity index. The regression model demonstrated that even after adjustment for age, comorbidities, disease severity, and presence of a DNR/DNI order, the presence of aspiration pneumonia was associated with higher mortality than CAP (odds ratio [OR]: $3.46, P<0.001$, Table 5). In this model, systolic blood pressure did not predict mortal-

\begin{tabular}{|c|c|c|}
\hline & Odds Ratio & $P$ Value \\
\hline Presence of aspiration pneumonia & $3.46(2.11-5.67)$ & $<0.001$ \\
\hline Age, y & $1.03(1.01-1.04)$ & $<0.001$ \\
\hline Confusion & $3.14(1.95-5.05)$ & $<0.001$ \\
\hline Blood urea nitrogen, mg/dL & $1.03(1.02-1.04)$ & $<0.001$ \\
\hline Respiratory rate, breaths/minute & $1.03(1.00-1.06)$ & 0.04 \\
\hline $\mathrm{PaO}_{2} / \mathrm{FiO}_{2}$ ratio, per $1 \mathrm{~mm} \mathrm{Hg}$ & $0.99(0.99-1.00)$ & $<0.001$ \\
\hline Moderate or severe liver disease & $9.21(3.16-26.86)$ & $<0.001$ \\
\hline Paraplegia/hemiplegia & $2.43(1.13-5.27)$ & 0.02 \\
\hline Diabetes with complications & $0.42(0.20-0.87)$ & 0.02 \\
\hline Leukocytosis & $4.47(2.27-8.82)$ & $<0.001$ \\
\hline DNR/DNI & $1.75(1.11-2.75)$ & 0.02 \\
\hline
\end{tabular}

NOTE: Initial model also included gender, presence of multilobar pneumonia, and all components of the CURB (Confusion, Uremia, Respiratory Rate, Blood Pressure) score and Charlson comorbidity index, and minor criteria for severe community-acquired pneumonia. Area under the curve of the final model $=0.87$. Odds ratios are followed by $95 \%$ confidence intervals in parentheses. Exclusion of DNR/DNI status did not significantly alter the regression model. Abbreviations: DNR/DNI, do not resuscitate/do not intubate. ity, and diabetes with complications was associated with decreased mortality.

\section{Microbiological Findings}

Blood cultures were performed at admission in $67.4 \%$ of aspiration-pneumonia patients, and a tracheal aspirate in half $(50.7 \%)$ of intubated patients with aspiration pneumonia. Organisms were recovered in 90 patients $(14.3 \%)$, although 41 of those patients had tracheal aspirates of organisms commonly thought to be nonpathogenic (nonpneumococcal alpha-hemolytic streptococcus, nonhemolytic streptococcus, diphtheroids, micrococci, coagulase negative staphylococccus). Tracheal aspirate was the most common method of recovering an organism (7.8\% of patients), followed by blood culture $(4.3 \%)$. Bronchoalveolar lavage, urinary antigen, and pleural fluid culture were less common $(1.3 \%, 1.1 \%, 0.3 \%$, respectively). The microbiologic results were grouped into: Staphylococcus aureus, Streptococcus pneumoniae, enteric bacilli, Haemophilus species, Neisseria species, Moraxella catarrhalis, and Pseudomonas aeruginosa (Figure 4). Comparing healthcare-associated with communityacquired aspiration pneumonia, healthcare-associated patients were more likely to have a confirmed infection with MRSA $(4.2 \%$ vs $1.4 \%, P=0.06)$ and enteric bacteria $(5.1 \%$ vs $1.6 \%, P=0.03)$. There were no other statistically significant differences in microbiologic recovery between the 2 groups. Antibiotics targeting anaerobic pathogens were administered in $28.7 \%$ of patients with aspiration pneumonia, with no correlation to the presence of healthcare-associated risks. Healthcare-associated patients were more likely to receive broad-spectrum antibiotics $(47.5 \%$ vs $32.5 \%, P<0.01)$ and MRSA coverage $(15.3 \%$ vs $5.7 \%, P<0.01)$ than patients with communityacquired aspiration pneumonia.

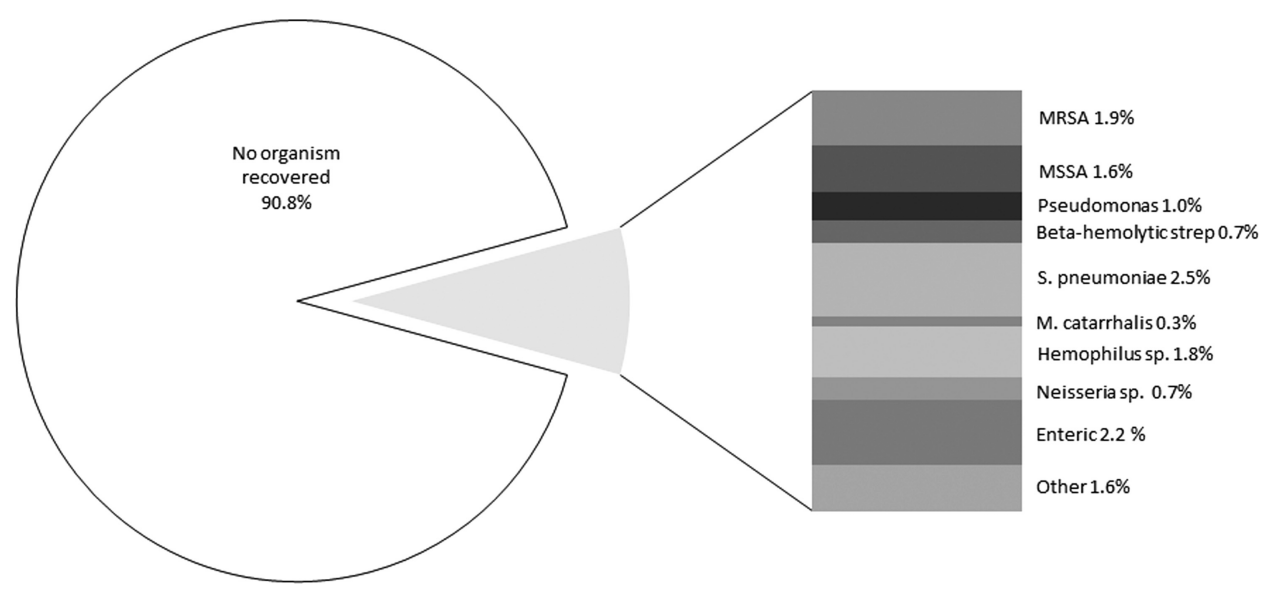

FIG. 4. Distribution of bacterial organism recovered from 628 patients with aspiration pneumonia. Percentages are expressed as a fraction of 628 patients. Note that the total exceeds $100 \%$ due to polymicrobial infection. Viral, fungal, and acid fast bacilli cultures were not routinely obtained and not included in this graphic. Other = Bacillus cereus (1), Serratia marcescens (1), Nocardia species (1), Acinetobacter bauminii (1), Capnocytophaga (1), Eikenella corrodens (1), Proteus (1), Saccharomyces cerevisiae (1). Abbreviations: M. catarrhalis, Moraxella catarrhalis; MRSA, methicillin-resistant Staphylococcus aureus; MSSA, methicillin-sensitive Staphylococcus aureus; S. pneumoniae, Streptococcus pneumoniae. 


\section{DISCUSSION}

Our study identifies a larger cohort of patients with aspiration pneumonia than previous studies. ${ }^{21-25}$ Patients with community-acquired aspiration pneumonia are older and more likely to die than CAP patients. They are more likely to be admitted to the hospital or ICU. Thirty-day mortality in this patient population was significantly underestimated by CURB-65 and eCURB, models developed and validated in CAP populations. ${ }^{9,26}$ This finding supports a prior study. ${ }^{27}$ It appears that a traditional prognostic model assessing mortality risk in the CAP patient does not apply to the aspiration-pneumonia patient. One reason for eCURB and CURB-65's poor utility in community-acquired aspiration pneumonia may be their reliance on objective clinical features rather than comorbidities, which may influence mortality to a greater degree in aspiration pneumonia.

This study has several limitations. There is no gold standard for the definition of aspiration pneumonia, and it is difficult to distinguish aspiration pneumonia from typical pneumonia. It is plausible that older patients with greater comorbidities are being designated as aspiration pneumonia. If this is the case, then aspiration pneumonia merely represents the end of the pneumonia spectrum with highest mortality risk, and it is no surprise that these patients fare poorly.

It appears that the hospitalist or emergency department physician implicitly appreciates that aspiration pneumonia has a higher mortality risk than predicted by traditional severity assessment. With such high mortality and morbidity, a patient presenting to the emergency room with aspiration pneumonia is almost always admitted to the hospital. Further work in this area should investigate other factors to improve prognostic modeling in patients with aspiration pneumonia, although the utility of such a model may be limited to determining ICU admission. Our data indicate that IDSA/ATS minor criteria for SCAP are not useful in predicting admission to the ICU in patients with aspiration pneumonia.

In this study, a DNR/DNI order was twice as common in the community-acquired aspiration pneumonia population than the CAP population. However, patients with community-acquired aspiration pneumonia and a DNR/DNI order were more than 3 times more likely to die than patients with CAP and a DNR/DNI order. Our regression model suggested that the presence of a DNR/DNI order was an independent predictor of mortality (OR: 1.75, $P<0.001$ ). Although a DNR/DNI order may correlate with the withholding or withdrawal of medical therapy, it is also a surrogate for increased age or comorbidities. ${ }^{28}$ In our study, however, the increased prevalence of DNR/DNI orders did not explain the poor mortality prediction of the eCURB or CURB-65, as exclusion of those patients did not significantly alter the AUCs in either the aspiration group or the CAP group.
Controversy exists regarding treatment of aspiration pneumonia. Historically, some have advocated for treatment of aspiration pneumonia with a regimen designed to cover anaerobic bacteria. ${ }^{29}$ This recommendation was based on early microbiologic studies that obtained the samples late in the course of illness, or other studies where the sample was obtained transtracheally, where oropharyngeal flora may contaminate the sample. ${ }^{30-32}$ Our clinically obtained microbiologic recovery of organisms was similar to the flora recovered in more recent CAP studies, in respect to both the incidence of pathogen recovery and the relative frequencies of recovered organisms. ${ }^{33,34}$ Our data do not support inferences regarding the prevalence of anaerobic infections, as the recovery of anaerobic organisms was limited to blood and pleural fluid cultures in this study, rather than techniques used in research settings that might have greater yield. As expected, patients with healthcare-associated risk factors trended toward increased incidence of MRSA. Given the similarity of the organisms recovered to those recovered in CAP, ${ }^{35}$ this study supports IDSA/ ATS recommendations that antibiotic therapy in aspiration pneumonia be similar to that of higher-risk CAP, with the addition of vancomycin or linezolid for MRSA coverage in patients with risk factors for healthcare-associated pneumonia. ${ }^{17}$

Our study is limited by its single-center retrospective design. However, beginning in 1995, the LDS Hospital emergency department initiated a standardized pneumonia therapy protocol and deployed electronic medical records, which prospectively recorded a wide array of clinical, therapeutic, and biometric data. Most data elements used in this analysis were routinely charted for clinical purposes in real time. Although the eCURB, CURB-65, and some comorbidities could be extracted electronically for each patient, it was not possible to calculate the pneumonia severity index score due to our inability to rigorously identify the necessary comorbid illness elements. Other comorbidities, not present in our model, may have been identified by the physician who makes a diagnosis of aspiration pneumonia. Our identification of swallow impairment is also methodologically limited. The decision to obtain a swallow study was clinical, usually occurring upon convalescence. Therefore, it is not possible to distinguish between antecedent oropharyngeal dysfunction and post-critical illness dysfunction.

Our definition of aspiration pneumonia required the treating physician to diagnose and code the patient as having aspiration pneumonia, followed by excluding patients more likely to have aspiration pneumonitis. Although we relied on ICD-9 codes to initially identify aspiration pneumonia, all patients in our database were confirmed by physician chart review. Our incidence of community-acquired aspiration pneumonia is congruent with other studies using different methodologies. ${ }^{1,36,37}$ Unfortunately, there is no standard and 
widely accepted definition for separating aspiration pneumonia from usual CAP. A younger and healthier patient who has developed pneumonia subsequent to aspiration may be more likely to be diagnosed with CAP, resulting in selection bias for older patients with greater comorbidities.

\section{CONCLUSION}

Patients diagnosed with aspiration pneumonia are older, have more comorbid conditions, and demonstrate greater disease severity and higher 30-day mortality than CAP patients. Mortality prediction using CURB-65 and eCURB in this population was poor, possibly due to a greater effect of comorbidities on mortality. The pneumonia severity index, which incorporates patient comorbidities, might perform better than the eCURB or CURB-65, and should be studied in aspiration pneumonia populations where comorbid illness information is prospectively collected. Further areas of study include creating an improved mortality prediction model for aspiration pneumonia that incorporates comorbid conditions, DNR/DNI status, and disease severity.

\section{Acknowledgements}

The authors acknowledge Al Jephson for database support, Yao Li for statistical analysis, and Anita Austin for help reviewing the medical records. Dr. Lanspa had full access to all of the data in the study and takes responsibility for the integrity of the data and the accuracy of the data analysis.

Disclosure: Preliminary versions of this work were presented as posters at the American Thoracic Society Meeting, Denver, Colorado, May 17, 2011. This study was supported by grants from the Intermountain Research and Medical Foundation. Dr. Brown is supported by a career development award from National Institute of General Medical Sciences (K23GM094465). Dr. Dean served on an advisory board for Merck, has been a paid consultant for Cerexa, and has received an investigator-initiated competitive grant from Pfizer for development of an electronic pneumonia decision support tool. All other authors report no relevant financial disclosures.

\section{References}

1. Torres A, Serra-Batlles J, Ferrer A, et al. Severe community-acquired pneumonia. Epidemiology and prognostic factors. Am Rev Respir Dis. 1991;144(2):312-318.

2. Koivula I, Sten M, Makela PH. Risk factors for pneumonia in the elderly. Am J Med. 1994;96(4):313-320.

3. Marik PE, Kaplan D. Aspiration pneumonia and dysphagia in the elderly. Chest. 2003;124(1):328-336.

4. Mylotte JM, Goodnough S, Naughton BJ. Pneumonia versus aspiration pneumonitis in nursing home residents: diagnosis and management. J Am Geriatr Soc. 2003;51(1):17-23.

5. Marik PE. Aspiration pneumonia: mixing apples with oranges and tangerines. Crit Care Med. 2004;32(5):1236; author reply 1236-1237.

6. Kozlow JH, Berenholtz SM, Garrett E, Dorman T, Pronovost PJ. Epidemiology and impact of aspiration pneumonia in patients undergoing surgery in Maryland, 1999-2000. Crit Care Med. 2003;31(7): 1930-1937.

7. Marik PE. Aspiration syndromes: aspiration pneumonia and pneumonitis. Hosp Pract (Minneap). 2010;38(1):35-42.

8. Marik PE. Aspiration pneumonitis and aspiration pneumonia. $N$ Engl J Med. 2001;344(9):665-671.

9. Jones BE, Jones J, Bewick T, et al. CURB-65 pneumonia severity assessment adapted for electronic decision support. Chest. 2011; 140(1):156-163.

10. Lim WS, van der Eerden MM, Laing R, et al. Defining community acquired pneumonia severity on presentation to hospital: an international derivation and validation study. Thorax. 2003;58(5):377-382.
11. Fine MJ, Hanusa BH, Lave JR, et al. Comparison of a disease-specific and a generic severity of illness measure for patients with communityacquired pneumonia. J Gen Intern Med. 1995;10(7):359-368.

12. Espana PP, Capelastegui A, Gorordo I, et al. Development and validation of a clinical prediction rule for severe community-acquired pneumonia. Am I Respir Crit Care Med. 2006;174(11):1249-1256.

13. Brown SM, Jones BE, Jephson AR, Dean NC. Validation of the Infectious Disease Society of America/American Thoracic Society 2007 guidelines for severe community-acquired pneumonia. Crit Care Med. 2009;37(12):3010-3016.

14. Guidelines for the management of adults with hospital-acquired, ventilator-associated, and healthcare-associated pneumonia. Am J Respir Crit Care Med. 2005;171(4):388-416.

15. Skolnick M. The Utah genealogical database: a resource for genetic epidemiology. In: Cairns JL, Skolnick M, eds. Banbury Report No 4; Cancer Incidence in Defined Populations. New York, NY: Cold Spring Harbor Laboratory; 1980:285-297.

16. Severinghaus JW. Simple, accurate equations for human blood O2 dissociation computations. I Appl Physiol. 1979;46(3):599-602.

17. Mandell LA, Wunderink RG, Anzueto A, et al. Infectious Diseases Society of America/American Thoracic Society consensus guidelines on the management of community-acquired pneumonia in adults. Clin Infect Dis. 2007;44(suppl 2):S27-S72.

18. Charlson M, Szatrowski TP, Peterson J, Gold J. Validation of a combined comorbidity index. J Clin Epidemiol. 1994;47(11):1245-1251.

19. Deyo RA, Cherkin DC, Ciol MA. Adapting a clinical comorbidity index for use with ICD-9-CM administrative databases. J Clin Epidemiol. 1992;45(6):613-619.

20. DeLong ER, DeLong DM, Clarke-Pearson DL. Comparing the areas under two or more correlated receiver operating characteristic curves: a nonparametric approach. Biometrics. 1988;44(3):837-845.

21. Feinberg MJ, Knebl J, Tully J. Prandial aspiration and pneumonia in an elderly population followed over 3 years. Dysphagia. 1996;11(2): 104-109.

22. Langmore SE, Skarupski KA, Park PS, Fries BE. Predictors of aspiration pneumonia in nursing home residents. Dysphagia. 2002;17(4): 298-307.

23. Terpenning MS, Taylor GW, Lopatin DE, Kerr CK, Dominguez BL, Loesche WJ. Aspiration pneumonia: dental and oral risk factors in an older veteran population. J Am Geriatr Soc. 2001;49(5):557-563.

24. El-Solh AA, Pietrantoni C, Bhat A, et al. Microbiology of severe aspiration pneumonia in institutionalized elderly. Am I Respir Crit Care Med. 2003;167(12):1650-1654.

25. Johnson ER, McKenzie SW, Sievers A. Aspiration pneumonia in stroke. Arch Phys Med Rehabil. 1993;74(9):973-976.

26. Capelastegui A, Espana PP, Quintana JM, et al. Validation of a predictive rule for the management of community-acquired pneumonia. Eur Respir J. 2006;27(1):151-157.

27. Heppner HJ, Sehlhoff B, Niklaus D, Pientka L, Thiem U. Pneumonia Severity Index (PSI), CURB-65, and mortality in hospitalized elderly patients with aspiration pneumonia [in German]. Zeitschrift fur Gerontologie und Geriatrie. 2011;44(4):229-234.

28. Bedell SE, Pelle D, Maher PL, Cleary PD. Do-not-resuscitate orders for critically ill patients in the hospital. How are they used and what is their impact? JAMA. 1986;256(2):233-237.

29. Gilbert DN, Moellering RC, Eliopoulos GM, Chambers HF, Saag MS. The Sanford Guide to Antimicrobial Therapy: 2010. 40th ed. Sperryville, VA: Antimicrobial Therapy, Inc.; 2009.

30. Bartlett JG, Gorbach SL, Finegold SM. The bacteriology of aspiration pneumonia. Am J Med. 1974;56(2):202-207.

31. Cesar L, Gonzalez C, Calia FM. Bacteriologic flora of aspirationinduced pulmonary infections. Arch Intern Med. 1975;135(5): 711-714.

32. Lorber B, Swenson RM. Bacteriology of aspiration pneumonia. A prospective study of community- and hospital-acquired cases. Ann Intern Med. 1974;81(3):329-331.

33. Marik PE, Careau P. The role of anaerobes in patients with ventilator-associated pneumonia and aspiration pneumonia: a prospective study. Chest. 1999;115(1):178-183.

34. Mier L, Dreyfuss D, Darchy B, et al. Is penicillin G an adequate initial treatment for aspiration pneumonia? A prospective evaluation using a protected specimen brush and quantitative cultures. Intensive Care Med. 1993;19(5):279-284.

35. Torres A, El-Ebiary M, Riquelme R, Ruiz M, Celis R. Communityacquired pneumonia in the elderly. Semin Respir Infect. 1999;14(2): 173-183.

36. Marrie TJ, Durant H, Kwan C. Nursing home-acquired pneumonia. A case-control study. I Am Geriatr Soc. 1986;34(10):697-702.

37. Moine P, Vercken JB, Chevret S, Chastang C, Gajdos P. Severe community-acquired pneumonia. Etiology, epidemiology, and prognosis factors. French Study Group for Community-Acquired Pneumonia in the Intensive Care Unit. Chest. 1994;105(5):1487-1495. 